\title{
Performance Analysis of OFDM, FBMC and UFMC Techniques
}

\author{
Firoz Ahmed \\ Information and Communication Engineering, University of Rajsahi, Rajsahi 6205, Bangladesh \\ fahmed@ru.ac.bd
}

Received: $10^{\text {th }}$ May 2021, Accepted: $13^{\text {th }}$ June 2021, Published: $30^{\text {th }}$ June 2021

\begin{abstract}
The project's major goal is to demonstrate that the FBMC and UFMC modulation techniques outperform the OFDM (Orthogonal Frequency Division Multiplexing) approaches. In this section, we compare certain essential parameters of three $5 \mathrm{G}$ modulation schemes to demonstrate that UFMC and FBMC outperform OFDM. Some parameters are taken into account for this, such as power spectrum density, spectral efficiency, and bit error rate.
\end{abstract}

Keywords: FBMC, UFMC, OFDM, PAPR

\section{Introduction}

Contact with Wireless: Communication networks may be cable or electronic, and the medium used for communication can be controlled or unguided. In Wire Communication, the path is a direct line, such as Non-axial Wires, Multimode fiber Wires and Fiber optic Lines, etc., which guides the signal to propagate from one point to another. This media type is called Guided Press. Wireless Communication, on the other hand, does not involve an external source, but spreads a pulse through air. Since space only really simply allows digital signal transmission system without any direction, The Media Unguided is a medium designed for wireless transmission. Wireless Communication is the fastest-growing and most vibrant field in communication technology. Wireless communication is a way of transferring information from one place to another, without the use of any links such as lines, cables or other external medium.

Normally, information is passed from the source to the receiver within a restricted distance in a communications network. Through the aid of Wireless Communications, the processor and recipient can be mounted anywhere from a few meters to a few thousand kilometers.. We live in a world of contact and wireless communication, and this is a vital aspect of our lives in particular. Some of the widely used wireless networking devices in our day-to-day life is: cell phones, Navigation receivers, remotes, Bluetooth audio and Wi-Fi etc.

\section{Need of Wireless Communication}

Since wireless communications can do much of the things that wireless communications have to do, why do we even need wireless technology? Flexibility is the main and essential advantage of wireless technology. Besides versatility, wireless communications often provides versatility and convenience of use, making it extremely common every day. With considerably high throughput performance, wireless communication like mobile telephony can be done anywhere and at any time. A further critical aspect is technology. The design and installation of infrastructure for wired communication systems is an costly and time-consuming task. The wireless network can be easily and costeffectively built. Wireless communication is a viable alternative in emergencies and remote places, where the installation of wired communications is complicated. 


\section{Literature Survey}

S.S.GHORPADEet.al[1] discusses implementation of OFDM transmitter and receiver in MATLAB. By dividing the broad fading channel of the wideband frequency into some narrow band flat fading channels, OFDM is intended to cope with the multipath propagation effect. OFDM uses the sub-carriers ' guard bands to keep them apart from each other to avoid the inter-symbol interference. The use of 64 subcontractors often needs less processing time and less complexity. Using power amplifiers, increase spectral efficiency and decrease peak to average power ratio, and several other methods can be employed to reduce PAPR such as clipping, scrambling, peak cancellation, etc. So, it addressed the problem of multipath fading.

The FBMC techniques used to increase the bits rate by reducing the guard interval and when cyclic prefix is not present in OFDM as needed as defined by Lalit Chettriet.al[2]. The future FBMC must improve efficiency and use the bandwidth to perfection. In FBMC OQAM post-processing requires two actions: the first is pattern multiplication.In addition, post-processing OQAM is conversion from real to complex, and a single complex symbol is valued in this symbol with two true valued.

But the conversion reduces sampling rates. The full model of FBMC is planned and simulated through the so-called Systemvue 2013.01 software. Before these, random bits are used in this model, as the input pulse can be transformed to the number. The Virtual Amplification model could be used to produce the digital amplification pulse of the baseband, which also includes the basic amplification systems. To this reason is used the modulation scheme 16QAM. Here the equalizer on the receiver side is used to solve the overlapping problems. The high PARP is the main issue Hanwanget.al[3] has implemented in all multicarrier communication systems. In the past, the methods used in OFDM to reduce PARP have been as follows: SLM, PTS scheme, and TR scheme, but these schemes were not useful to FBMC because of the different FBMC structure. A hybrid scheme for FBMC / OQAM signals to lower the PARP and that depends on the multi data block approaches PTS and TR. This is a very successful method in canceling signal peaks.

Behrouz Farhang-Boroujeny et.al suggested the idea of the various multicarrier techniques[4]. Secondly, in wireless communication, the OFDM (orthogonal division frequency multiplexing) suggested addressing ISI. It splits the available bandwidth into several subcarrier firms. Prior to the OFDM method, there was another method for synthesizing and analyzing multi-carrier signals, using filter banks.

It has been often proposed to modulate CMT and VSB with filter banks. The provided signal bandwidth range is divided into two classes in VSB (Vestigial Side-Band Modulation), i.e. baseband signal. The first part of the baseband signal is used for the modulation of a complex sine wave at a positive frequency and the second part of the baseband signal is used for the modulation of complex sine wave at a negative frequency. The multicarrier technique is useful for compromising with small frequency channels, where the gain variations occur across the frequency band which can cause ISI and ICI. Channel equalization mechanism is used to overcome this problem with multicarrier system.

Centralized non-equalization is being used to balance the network downside between multiple users with reduced complexity and network cost. So the FBMC works with pre-equalization stronger than OFDM, because FBMC uses prototype filters to evaluate the moment-frequency window for each subcarrier. You will lower the undesirable side lobes in doing so. It helps the system to incorporate asynchronous CCs inside without involving numerous clients, suppliers and locations. The MMSE is used to get the pre- equalisation here. The shortcoming of use of FBMC is that there is often an inherent theoretical conflict between adjacent subcarriers and identifiers before preequalization. This makes it difficult to estimate networks by preamble definition. $\mathrm{Mu} \mathrm{Xu}$ et.al[5] proposed a comparison of the efficiency and performance of FBMC and OFDM with centralized pre-equalization and without centralized pre-equalization. 


\section{OFDM}

Orthogonal Frequency Division Multiplexing is a type of message waveform or amplification offering several major advantages for data connections. Appropriately, many of the modern broad spectrum and high data rate wireless networks like Wi-Fi, cellular phones and many others use OFDM, the Orthogonal Frequency Division Multiplexing. The reality that OFDM uses a huge number of carriers, each holding low baud rate data, implies that it is extremely sensitive to specific fading, interruption, and spanning tree effects, as well as delivering a high level of spectral efficiency. OFDM's features are used in many data transfer rate wireless networks.

\section{Advantages}

- Strength to interruption: network intervention can be restricted in bandwidth and therefore does not affect all post-channels. It does not mean the proof is totally lost.

- Spectrum efficiency: The main benefit of using tight-spaced alternating subcarriers for OFDM is that it makes efficient use of the usable bandwidth.

- ISI resilient: Another benefit of OFDM is that the interaction between the symbols and the interframes is very stable. This leads in small data rate on each of the post-channels.

- Simplified channel equalization: One of the difficulties with CDMA devices was the network equalizing complexity which had to be applied around the network. An advantage of OFDM is that it may be simpler to equalize the networks, using many post-channels.

\section{Disadvantages}

- High maximum-to-average power ratio: an OFDM signal has a noise-like amplitude variation and a fairly wide dynamic range or maximum-to-average ratio. It affects the RF amplifier's output as the amplifiers should be static to accommodate the large amplification fluctuations so that even the amplifier could not pirate with a high level of efficiency.

- Carrier-sensitive offset and drift: OFDM's other drawback is its sensitivity to offsets and drift at carrier frequencies. Second-carrier systems less sensitive.

OFDM is a multi-carrier transmission system in which the frequency band in a variety of sub-channels is distinct. Usual multiplexing methods require a variety of filters to avoid interference between sub-carriers and nonoverlapping must be established with a minimum separation of frequencies. On the other hand, OFDM uses signal processing techniques that also mitigate this problem, since the subcarriers are orthogonal in nature and remove the need for multiple filters. An OFDM network consists of a receiver and a transmitter. The various modulation techniques map the signal to a suitable constellation. This serial data is then translated into a parallel stream of data on which OFDM is executed. It is composed of $\mathrm{N}$ sub carriers that bear the symbols. An IFFT block is involved in an OFDM transmitter.

$$
f(n)=\sum_{K=0}^{N-1} \exp \left(\frac{j 2 \pi k n}{N}\right)
$$

To minimize ISI, the cyclic prefix is appended to the output. This is then translated to a serial output that passes through the respective tube. The data is transformed to parallel input at the processor, and the cyclic prefix is removed. This then comes under FFT. The frequency domain signal in subcarrier receiving kth is expressed as

$$
F(K)=\sum_{n=0}^{N-1} f(n) \exp \left(-\frac{-j 2 \pi k n}{N}\right)
$$




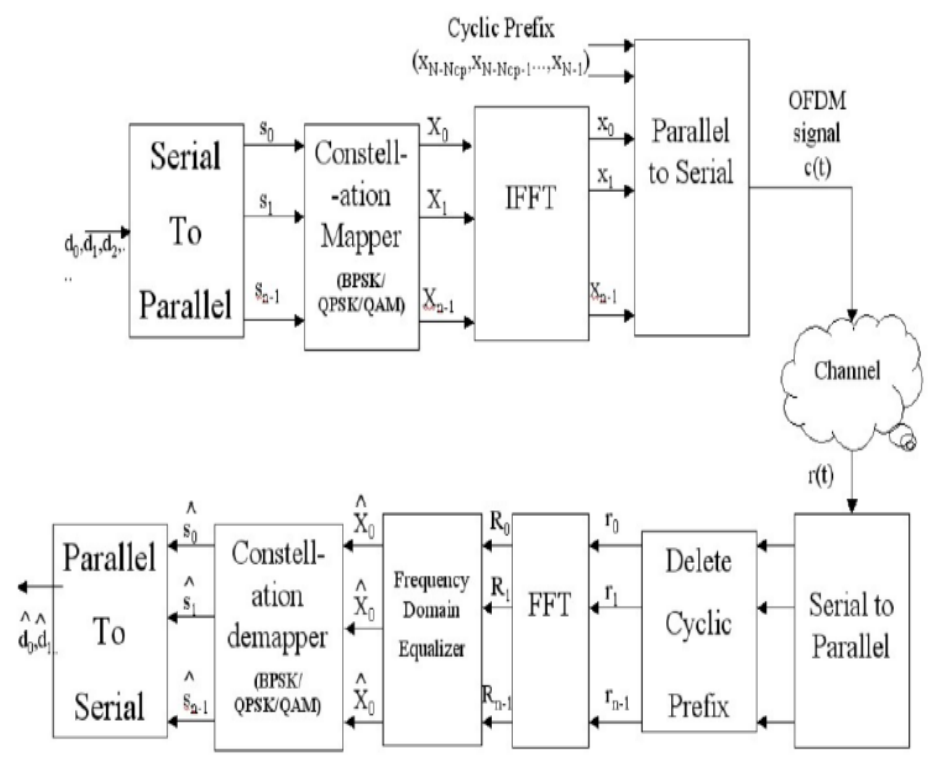

Fig 1: OFDM model

\section{FBMC}

This is a form of multi-carrier amplification originating from within OFDM. This is an OFDM innovation that aims to solve some of the problems but that is at the cost of better signal processing. It allows much greater use of the limited network capacity, and can provide larger data rates within a limited radio frequency spectrum, i.e. it has higher bandwidth efficiency. It aims to fix some of the limitations the orthogonal frequency group, OFDM, has encountered with multipathing.

Some of main disadvantages are the use of what has been called a cyclic prefix is needed by OFDM. The cyclic prefix is essentially a duplication of part of the transmitted OFDM symbol which is added at the beginning of this next. Such efficiency enhances the transmitting capacity and thereby reduces excess power.

OFDM's disadvantage is that the spectrum location of the subcarriers is small; causing problems of spectral leaking and interfering with semi-synchronized signal. The bank's Filter multicarrier is an OFDM invention. Use of filter banks added, typically using these methods for optical image processing. In an OFDM scheme, sidelobes stretched out on the other side as carries were amplified. The filters are used to remove these effects, using a filter bank system, and thus a much safer carrier. Systems that employ FBMC amplification are more specific than OFDM. That benefits from the exchange of filter banks

\section{FBMC advantages}

- FBMC will deliver a more powerful and flexible spectrum program.

- There is no need for the cyclic prefix, CP required for OFDM, thus freeing up more room for real data.

- Offering reliable narrowband jammers

\section{FBMC limitations}

- The architecture of FBMC's large bandwidth and high dynamic range systems creates soem-significant challenges for RF growth.

- This is much more complicated than OFDM-it imposes overload symbols in the filter banks in time domain overlap UFMC 
FBMC filters any sub-carrier that modulates a signal in a multi-carrier network. The model device is that used by the negative frequencies carrier and the passband's basis for other filters. The filters are defined by the overlap factor, $\mathrm{K}$, which would be the numbers of multi-carrier symbols overlapping within the domain. User can choose the order of the prototype filter. Present FBMC implementation uses Frequency Spreading. It uses an IFFT N*K length with N/2 delay-overlapped symbols, where $\mathrm{N}$ is the subcarrier number. This design choice makes FBMC quick to test and compared to other modification processes.

(OQAM) is being used to achieve optimum efficiency. The rational and irrational parts of a large data symbol are also not equally represented, since half the length of the symbol delays the imaginary part. The Detect-end processing is shown in the diagram below.

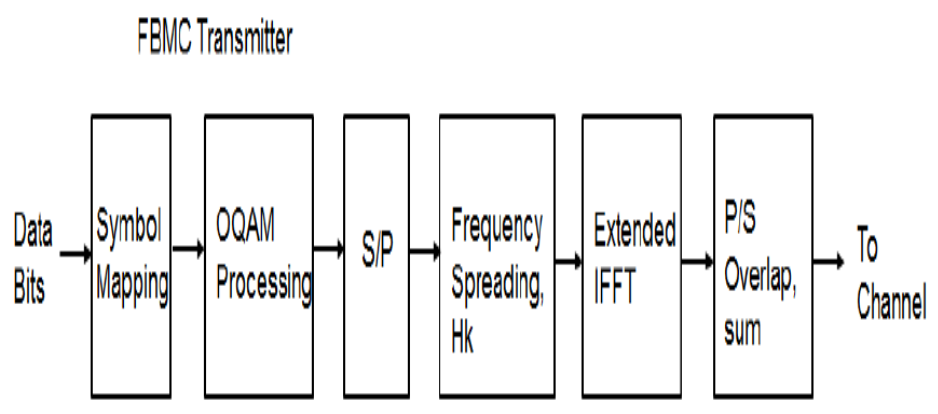

Fig 2: FBMC Transmitter

The example introduces a simple FBMC demodulator in the absence of a path, and measures the BER for the chosen specification. The processing requires matched filtering to form the data symbols collected, followed by separation from the OQAM. These are demapped to parts, and the resultant bit error rate is set. Linear multi-tap equalizers can be used to mitigate the effects of wavelength-selective flickering in the presence of a signal. By offering higher spectral performance, FBMC is deemed advantageous compared to OFDM. The processing of the receive-end is shown in diagram below.



Fig 3: FBMC Receiver 


\section{UFMC}

Like other 5G Waveform candidates, the filtering method is also based on UFMC. The differentiator is how the filter is applied, and what kind. In the case of FBMC, it packs several consecutive subcarries into a category named' subband' and applies the filter as outlined below to each subband. How many subcarriages a subband should have belonged to is a design specification. The more subcarriers in a subband, the lower the baseband processing load, but the weaker the final output when you add more subcarriers into one subband.

UFMC is often used as a generalization of Filtering OFDM and FBMC modulations. The complete band is filtering via filtered OFDM and independent subcarriers are filtering via FBMC while subcarrier groups (subbands) are filtered via UFMC. This subcarrier classification lets us get to the filter length (as opposed to FBMC). UFMC can tend to use QAM that works with established MIMO schemes as well.

Total subcarrier $(\mathrm{N})$ band are split into subbands. Each subband has a fixed number of subcarriers for a defined transmission, and not all subbands need to be employed. An N -pt IFFT is calculated of each subband, adding zeros for both the unassigned carries. A length filter L filters each subband, and summarizes the responses from the different subbands. Filtering is performed to reduce the spectral emissions from outside the band. Various filters may be built per subband but the same filter is used for each subband in this example.

To filter performance IFFT by subband window for user defined sidelobe attenuation, a Chebyshev window is being used to filter output IFFT by subband with parameterized side lobe attenuation.

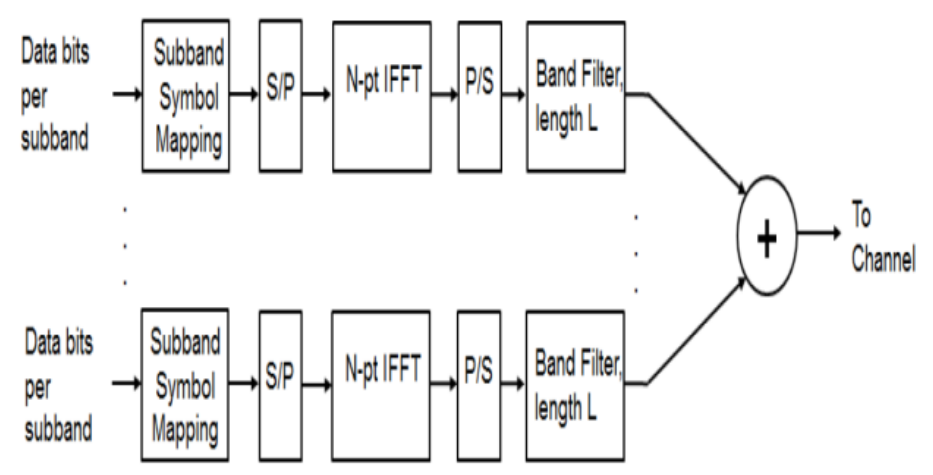

Fig 4: UFMC Transmitter

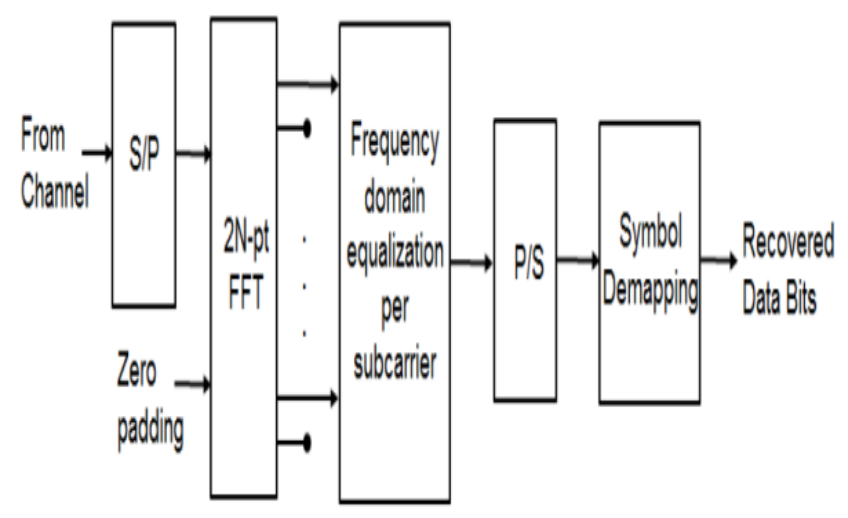

Fig 5: UFMC Receiver 
By providing higher spectral efficiency UFMC is considered advantageous compared with OFDM. The subband filtering expands the window of time to next power-of-two range for the FFT operation. The alternate frequency value matches a subcarrier's main lobe. In normal cases, per-subcarrier equalization is being used to equalize the reciprocal effect and filtering of subbands of the signal. In this case, only the subband filter is equalized since no channel effects are modelled.

\section{Software Description}

\section{MATLAB Product Description}

Millions of engineers and scientists worldwide use MATLAB ${ }^{\circledR}$ to analyze and improve the technologies and products that are changing our climate. MATLAB is involved in vehicle protection systems, interplanetary satellites, health monitoring devices, wireless LTE networks and smart power grids. It is used in the fields of machine learning, signal processing, image processing, computer vision, communication, computer finance, design control, robotics, and more. The MATLAB method is designed to solve the technological and scientific problems. The MATLAB language based on matrixes is the most normal way for computational physics to convey itself in the world. Built-in graphics make the visualization and insight from the data easy. A large library of pre-constructed toolboxes helps you to get started with algorithms that are important for your domain right away. The desktop environment is an invitation to play, explore, and discover. The methods and functionality of these MATLAB are all rigorously tested and built to work together

\section{Key Specifications}

- Big-level engineering language programming

-Workspace framework built for iterative testing, design and problem-solving

- Data modeling graphics and customized plot creation tools - Curve fitting applications, data detection, signal analysis, control unit tuning and several other tasks

- Add-on toolboxes for a broad variety of development tasks.

\section{Results}

Technologies like OFDM, FBMC, and UFMC were investigated in the project based on parameters such as random number generator, number of subbands, SNR, sidelobe attenuation, subband height, filter length, subband offset value with the Offset Quadrature A modulation technique.

In this first stage the power spectral density is compared between OFDM and FBMC as shown in fig 6. It is clearly evident here that FBMC, as compared to OFDM, restores optimum power spectral density. The same is true for Table 1.

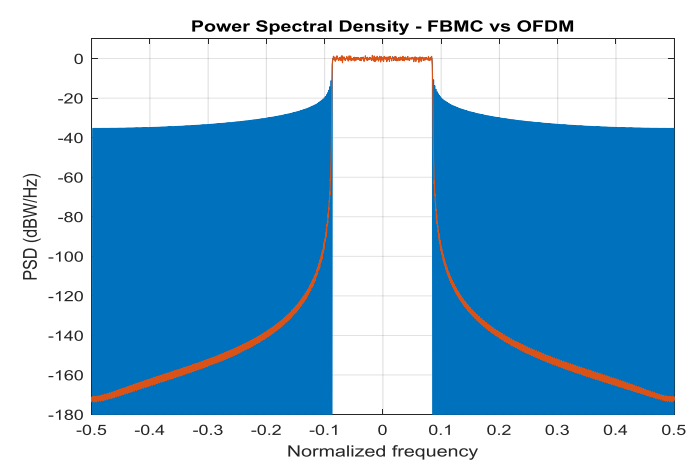

Fig 6: PSD -FBMC Vs OFDM 


\section{Table 1: PSD -FBMC Vs OFDM}

\begin{tabular}{|c|c|c|}
\hline $\begin{array}{l}\text { Normalized } \\
\text { Frequency }\end{array}$ & FBMC & OFDM \\
\hline-0.5 & -174 & -34 \\
\hline-0.4 & -168 & -34 \\
\hline-0.3 & -158 & -32 \\
\hline-0.2 & -142 & -30 \\
\hline-0.1 & 0 & -18 \\
\hline 0 & 0 & - \\
\hline 0.1 & 0 & -18 \\
\hline 0.2 & -142 & -30 \\
\hline 0.3 & -158 & -32 \\
\hline 0.4 & -168 & -34 \\
\hline 0.5 & -174 & -34 \\
\hline
\end{tabular}

The PSD between OFDM and UFMC is compared in this second level, as shown in fig 7. The UFMC restores optimum power spectral density as opposed to OFDM is clearly evident here. The same can be observed in the table 2 .

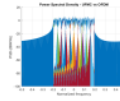

Fig 7: Power Spectral Density-UFMC vs OFDM 
Table 2: Power Spectral Density-UFMC vs OFDM

\begin{tabular}{|c|c|c|}
\hline Normalized Frequency & UFMC & OFDM \\
\hline-0.5 & - & -32 \\
\hline-0.4 & - & -30 \\
\hline-0.3 & - & -28 \\
\hline-0.2 & 0 & -8 \\
\hline-0.1 & 2 & - \\
\hline 0 & 4 & - \\
\hline 0.1 & 4 & - \\
\hline 0.2 & 6 & -16 \\
\hline 0.3 & - & -30 \\
\hline 0.4 & - & -30 \\
\hline 0.5 & - & -30 \\
\hline
\end{tabular}

Three methods are compared to later spectral efficiency, as shown in Fig 8. It is found that the values between OFDM and UFMC overlap where the incremental rise in FBMC can be found. The same can be seen in table 3 .

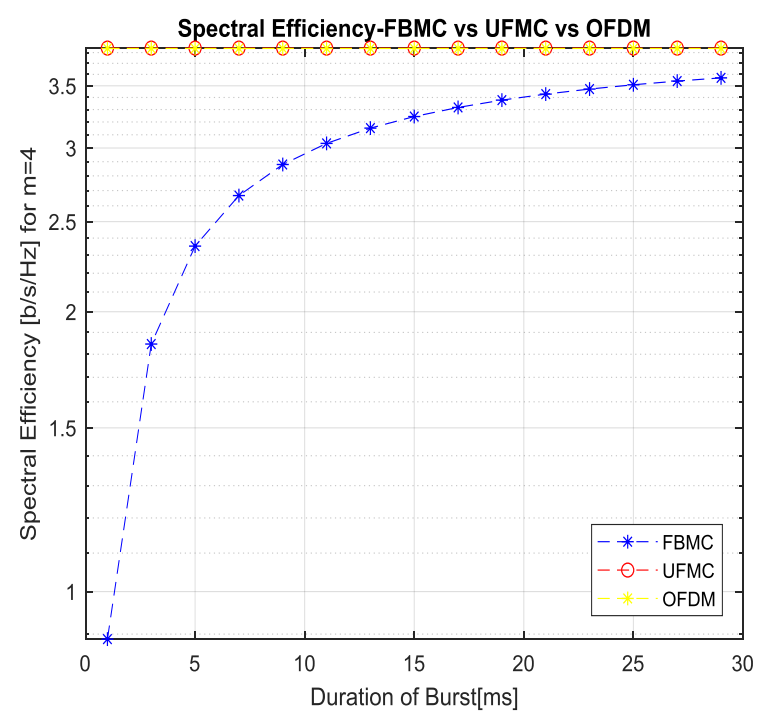

Fig 8: Spectral Efficiency-FBMC vs UFMC vs OFDM 


\section{Table 3: Spectral Efficiency-FBMC vs UFMC vs OFDM}

\begin{tabular}{|c|c|c|c|}
\hline \multirow{2}{*}{$\begin{array}{c}\text { Duration } \\
\text { of } \\
\text { Burst(ms) }\end{array}$} & \multicolumn{3}{|c|}{ Spectral Efficiency } \\
\cline { 2 - 4 } & FBMC & UFMC & OFDM \\
\hline 1 & 0 & 4 & 4 \\
\hline 3 & 1.85 & 4 & 4 \\
\hline 5 & 2.4 & & 4 \\
\hline 7 & 2.7 & & 4 \\
\hline 9 & 2.8 & 4 & 4 \\
\hline 11 & 3 & 4 & 4 \\
\hline 13 & 3.15 & 4 & 4 \\
\hline 15 & 3.2 & 4 & 4 \\
\hline 17 & 3.3 & 4 & 4 \\
\hline 19 & 3.4 & 4 & 4 \\
\hline 21 & 3.45 & 4 & 4 \\
\hline 23 & 3.5 & 4 & 4 \\
\hline 25 & 3.55 & 4 & 4 \\
\hline 27 & 3.6 & 4 & 4 \\
\hline 29 & 3.6 & 4 & 4 \\
\hline & & & \\
\hline
\end{tabular}

The analysis is then conducted to measure the signal to noise ratio for the three approaches to the bit error rate as shown in figures 9, 10 and 11. These graphs are tabulated in the table 4 which clearly shows FBMC is having an upper hand

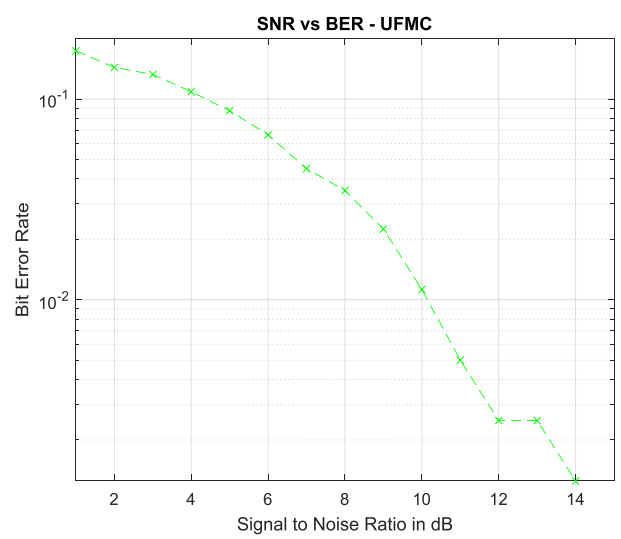

Fig 9: SNR vs BER-OFDM 


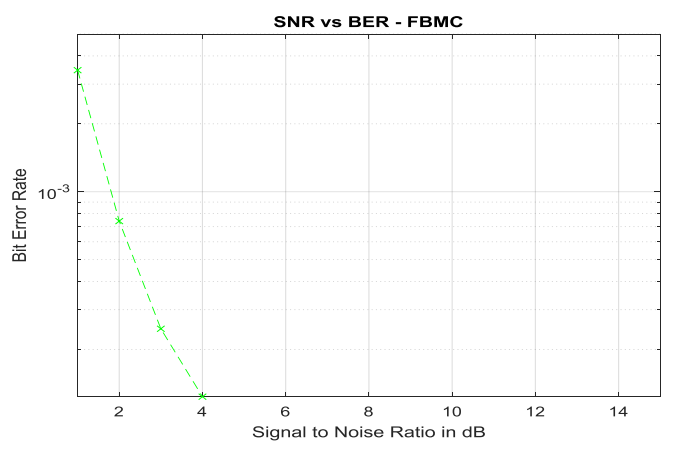

Fig 10: SNR vs BER-FBMC

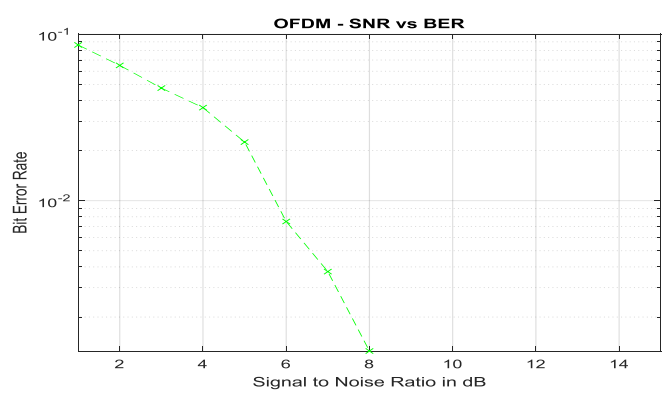

Fig 11: SNR vs BER-UFMC

Table 4: SNR vs BER of FBMC, UFMC and OFDM

\begin{tabular}{|c|c|c|c|}
\hline $\begin{array}{c}\text { SNR } \\
\text { in } \\
\mathrm{db}\end{array}$ & $\begin{array}{c}\text { BER } \\
\text { of } \\
\text { FBMC }\end{array}$ & $\begin{array}{c}\text { BER } \\
\text { of } \\
\text { UFMC }\end{array}$ & $\begin{array}{c}\text { BER } \\
\text { of } \\
\text { OFDM }\end{array}$ \\
\hline 1 & 0.0034 & 0.173 & 0.086 \\
\hline 2 & 0.0007 & 0.143 & 0.065 \\
\hline 3 & 0.0002 & 0.132 & 0.047 \\
\hline 4 & 0.0001 & 0.108 & 0.036 \\
\hline 5 & 0 & 0.087 & 0.022 \\
\hline 6 & 0 & 0.066 & 0.007 \\
\hline 7 & 0 & 0.045 & 0.003 \\
\hline 8 & 0 & 0.035 & 0.001 \\
\hline 9 & 0 & 0.025 & 0 \\
\hline 10 & 0 & 0.011 & 0 \\
\hline 11 & 0 & 0.005 & 0 \\
\hline 12 & 0 & 0.002 & 0 \\
\hline 13 & 0 & 0.002 & 0 \\
\hline 14 & 0 & 0.001 & 0 \\
\hline 15 & 0 & 0 & 0 \\
\hline
\end{tabular}




\section{Conclusion}

Here, we compared the three modulation techniques OFDM, FBMC and UFMC by considering some parameters. By looking at the graphs we could say FBMC, UFMC is far better than the OFDM. In this respect, different modulation schemes can also be established through wireless communications.

\section{References}

[1] S.S.Ghorpade, and Mrs.S.V.Sankpal, "Cosine Behavior of OFDM System Using MATLAB Simulation”, (IJITR) International Journal Of Innovative Technology And Research, Vol. 1, Issue 3, pp. 249-252, May. 2013.

[2] L.Chettri, Rabindranathbera, and D.Bhaskar, "Design and simulation of OQAM based filter bank multicarrier (FBMC) for 5G wireless communication systems", Proceedings of 24th IRF International Conference on Image Processing, Bengaluru, India, pp. 1-6, May 2016.

[3] Hanwang, X.Wang, L.Xu, and W.Du, "Hybrid PAPR Reduction Scheme for FBMC/OQAM Systems Based on Multi Data Block PTS and TR Methods”, IEEE Access, Vol. 4, pp. 4761-4768, Sept. 2016.

[4] B.Farhang-Boroujeny and C.H.(George)Yuen, "Cosine Modulated and Offset QAM Filter Bank Multicarrier Techniques: A Continuous-Time Prospect', EURASIP Journal on Advances in Signal Processing, Vol. 2010, Issue 6, pp. 1-16, Dec. 2014.

[5] M.Xu, J.Zhang, F.Lu, J.Wang, L.Cheng, H.J.Cho, M.I.Khalil, "FBMC in Next-Generation Mobile Fronthaul Networks with Centralized Pre- Equalization”, IEEE Photonics Technology Letters, Vol. 28, Issue 18, pp. 1912 1915, Sept. 2016.

[6]Haojie Yuan, Erlei Li, Fanchang Zeng. Filter Design for Suppressing Sidelobe Interference in NC-OFDM System [C]. 2018 International Symposium on Communication Engineering \& Computer Science (CECS 2018), 2018, July, $72-77$.

[7]F. Schaich and T. Wild. Waveform contenders for 5G OFDM vs. FBMC vs. UFMC Communications, Control and Signal Processing (ISCCSP) [C]. 2014 6th International Symposium on, Athens, 2014, pp. 457-460.

[8] Mei Ling Li, An Hong Wang and Gang Fei Wang. An analysis Model for OFDMA based intercell interference [J]. Journal of Information Hiding and Multimedia Signal Processing. 2016, 659-673. 